\title{
Smallest lithographic marks generated by optical focusing systems
}

\author{
T. Grosjean, D. Courjon, and C. Bainier \\ Département d'Optique P.M. Duffieux, Institut FEMTO-ST, UMR CNRS 6174, Université de Franche-Comté, \\ 16 route de Gray, 25030 Besançon Cedex, France
}

\begin{abstract}
We show that the combination of Bessel beams and photosensitive materials exhibiting polarization filtering properties allows one to reach the smallest mark that can be lithographically generated by focusing systems. This property is of interest in current optical data storage techniques.
\end{abstract}

In the context of system miniaturization, one of the major technological challenges is the improvement of data storage capacities. Optical data storage takes a growing part in this demand (compact and digital versatile discs). Among the solutions proposed for the next five to ten years to enhance optical disc capacity, ${ }^{1}$ one consists of reducing the spot size generated by the focusing device. Recently it has been shown that spot size minimization at focus, called focusing optimization, leads to the generation of the unfocused Bessel beam. ${ }^{2}$ This conclusion has been drawn by rigorously analyzing focused fields as the coherent combination of Bessel beams. It has been then demonstrated that, in practice, ultimate focal spots can be reached in a dielectric medium, such as photosensitive polymer, whose index of refraction matches the numerical aperture (NA) of the focusing system. ${ }^{3}$ Despite high interest in this solution for data storage (it should produce the smallest lithographic marks), it appears to be quite restrictive for practical applications. The NA cannot be arbitrarily chosen, since it has to be equal to the real part of the optical index of the photoresist. At the same time, it has been shown that azo-dye polymers develop a nonlinear behavior with respect to the vectorial light field during exposure. ${ }^{4,5}$ It has been pointed out that the surface relief modulation induced during exposure is proportional to the square modulus of the electric field component, which is perpendicular to the film interfaces (longitudinal field).

In this Letter, it will be shown that such a polarization filtering (PF) property of photoresists has strong consequences in lithography and data storage performances, since it provides an alternative way to achieve the smallest lithographic mark by focusing in the linear optical regime. This solution has the advantage of overcoming the problems related to the NA-to-photoresist optical index matching of the first solution. The smallest mark can be obtained whatever the NA. Let us note that a solution for improving the resolution has been proposed in Ref. 6, where the author speculates about the capacity of a particular medium to selectively record the longitudinal field component.

To understand how the PF phenomenon optimizes the surface patterning performance, it is necessary to analyze the confining process of the field distribu- tions that are produced by conical optics, i.e., Bessel beams. ${ }^{7,8}$ For this purpose, let $E_{L}$ and $E_{T}$ denote the electric field components that are perpendicular and parallel to the photosensitive layer interfaces. They will be called longitudinal and transverse components, respectively. From Ref. 3, the light spots generated by Bessel beams are described either by $E_{L}$ or by $E_{T}$ in the field expression. Therefore it turns out that $\left|E_{L}\right|^{2}$ is responsible for the light spot in radial polarization, whereas the light spot is due to $\left|E_{T}\right|^{2}$ in linear, elliptic, and circular polarizations. Because focused beams can be seen as a coherent superposition of Bessel beams, this analysis can be extended to focused beams. Moreover, when $E_{L}\left(E_{T}\right)$ is responsible for light confinement, $E_{T}\left(E_{L}\right)$ tends to widen the spot. Such a complementarity between $E_{L}$ and $E_{T}$ is intrinsic to the axial symmetry properties of focused beams and is not dictated by polarization.

Photochemical reaction of PF polymers is triggered by only one component of the 3D impinging light field during exposure. If the polarization is properly chosen for the Bessel beam, it is possible to make this sample interact with only the component that is responsible for the light spot $\left(E_{T}\right.$ or $\left.E_{L}\right)$. In this case, the photoresist filters out the undesirable other field components that tend to broaden the confinement zone. In this case, the material by itself generates the smallest mark that can be obtained by focusing.

Since the intensity of the smallest focal spot is described by function $J_{0}^{2}(2 \pi N A r / \lambda)$ ( $r$ is the radial spatial coordinate) and the surface topography is supposed to be proportional to this optimized spot, we can deduce a general equation defining the FWHM of the smallest mark profile as a function of NA and $\lambda$. We have

$$
\mathrm{FWHM}=0.36 \lambda / \mathrm{NA} .
$$

It is noteworthy that the mark size is evaluated with a trivial scalar theory, despite the vectorial bases of this work. For usual focused beams, lithographic patterns are defined by Airy functions (combination of Bessel functions $J_{0}$ ). Therefore, the relation defining the mark size can be easily found by deriving the well-known Rayleigh diffraction formula. The PF property newly discovered for PMMA-DR $1^{4,5}$ shows that it can generate the smallest lithographic mark 
when illuminated by a radially polarized Bessel beam ( $E_{T}$ is filtered out).

The PMMA-DR1 emulsion consists of a polymer matrix (PMMA) functionalized with azobenzene molecules (DR1). During exposure to light in its absorption band ${ }^{9}(400-600 \mathrm{~nm})$, direct surface modulations are created by means of repeated photoisomerization cycles of the azobenzene molecules. ${ }^{10,11}$

To validate our approach, a radially polarized Bessel beam is recorded into the layer of PMMA-DR1 with a technique borrowed from photography (beam snapshot). The Bessel beam is generated by transmitting an argon laser beam $(\lambda=514 \mathrm{~nm})$ through an axicon whose NA is equal to 0.67 . Let us note that finer patterning can be easily achieved by reducing the wavelength or/and enhancing the NA. Radial polarization is created by means of an optical fiber device. ${ }^{12}$ The PMMA-DR1 layer is deposited by spin coating onto a microscope cover glass so that the average film thickness is about $100 \mathrm{~nm}$. Bessel beam power and exposure duration are set to $32 \mathrm{~mW} \mathrm{~cm}^{-2}$ and $0.5 \mathrm{~s}$, respectively, leading to a maximum photon dose inside the polymer of $20 \mathrm{~J} \mathrm{~cm}^{-2}$ (calculations not detailed here). The topography of the surface imprint is measured with a home-built near-field microscope combined with a well-known shear-force device. ${ }^{13}$

To our knowledge, no photosensitive emulsions are sensitive to the transverse components of the illuminating field. However, it has been demonstrated that bare fiber sharp tips used in collection mode [scanning tunneling optical microscopy (STOM) configuration] exhibit such filtering properties ${ }^{14}\left(E_{L}\right.$ is filtered out). Therefore the image obtained by scanning the circularly polarized Bessel beam with these probes can give direct information about the characteristics of the mark generated by $E_{T}$-sensitive emulsions. The bare fiber tips used in this study are fabricated with the well-known heating-pulling technique. Circular polarization is obtained by making a linearly polarized laser beam passing through a quarterwavelength plate properly oriented.

To estimate the filtering effect on the mark size, we have compared the transverse intensity distributions of the radially polarized focused beam (RPFB) at focus [Fig. 1(a)], of the radially polarized Bessel beam (RPBB) [Fig. 1(b)], and the topography of the surface pattern generated in PMMA-DR1 by the RPBB [Fig. 1(c)]. The field expressions used to simulate the RPFB and RPBB can be found in Refs. 15 and 16.

From the intensity profiles of Fig. 1(d), we see that the FWHM of the RPFB, which equals $1.86 \lambda$ $(956 \mathrm{~nm})$, is reduced to $0.89 \lambda(457 \mathrm{~nm})$ in the case of RPBB. The recorded pattern exhibits a FWHM of $0.62 \lambda(322 \mathrm{~nm})$. This experimental value is larger than the theoretical prediction of $0.54 \lambda(276 \mathrm{~nm})$ given in Eq. (1). The discrepancy can be explained either by the influence of the scanning probe used for topography acquisition, which can slightly enlarge the central dot, or by the azo-dye polymer optical response. Nevertheless, the size of the experimental mark is threefold and 1.43-fold smaller than the theoretical RPFB and RPBB spot sizes, respectively. Since the real part of the optical index of

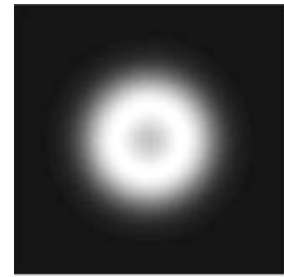

(a)

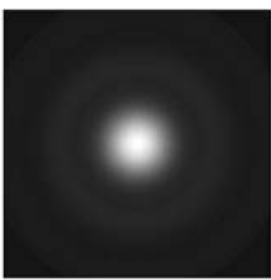

(b)

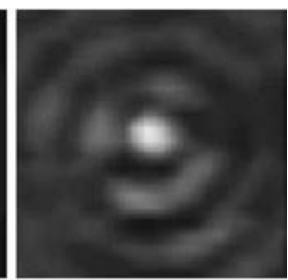

(c)



(d)

Fig. 1. (a) Intensity of the RPFB at focus (simulation); (b) transverse intenstiy of the RPBB (simulation); (c) surface deformation of the PMMA-DR1 layer (experiments). Image size, $2 \mu \mathrm{m} \times 2 \mu \mathrm{m}$. (d) Profiles of (a) (dotted curve), (b) (dashed curve), (c) (solid curve), and of the smallest mark (dashed-dotted curve, simulation).

PMMA-DR $1^{17}$ (around 1.6) is far from the value of $\mathrm{NA}=0.67$, the fact that the mark size is smaller than the spot size of the corresponding incident RPBB is due to $\mathrm{PF}$ properties of the photosensitive film. It is not induced by refraction effects on the radially polarized Bessel beam that propagates into the dielectric layer. ${ }^{3}$

We also note that the confinement capacity of the $\mathrm{RPBB}$ in air, which corresponds to the expeximentally measured mark size, requires the use of a conical objective of NA around 0.99 (instead of 0.67 in our case). The price to pay for the maximum mark shrinkage is the presence of a parasitic surface modulation surrounding the central dot, which is due to the lateral fringes of the incident Bessel beam. Nevertheless, by adjusting the Bessel beam power and/or exposure duration, this undesirable surface relief can be strongly reduced as shown in Fig. 2 . The photoinduced topography displayed in Fig. 2(a) is obtained with a time duration twofold the time duration used in the case of Fig. 2(b), with a constant value of the incident power. The asymmetric pattern shown in Fig. 2(b) can be explained by slight optical nonlinearities of the surface deformation process.

In Fig. 3 are compared the light spots of the circularly polarized focused beam (CPFB) [Fig. 3(a)], of the circularly polarized Bessel beam (CPBB) [Fig. $3(\mathrm{~b})$ ], and the experimental simulation of the mark generated with the CPBB [Fig. 3(c)]. 


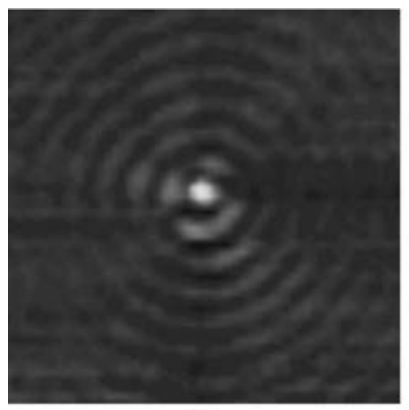

(a)

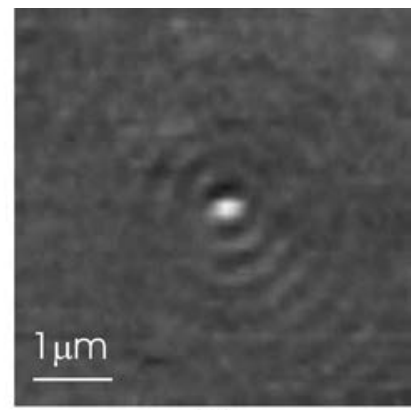

(b)

Fig. 2. Topography of the surface modulation produced by a RPBB. The photon dose in (b) is twofold smaller than the one in (a).

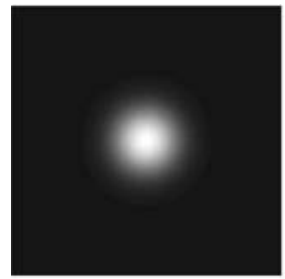

(a)

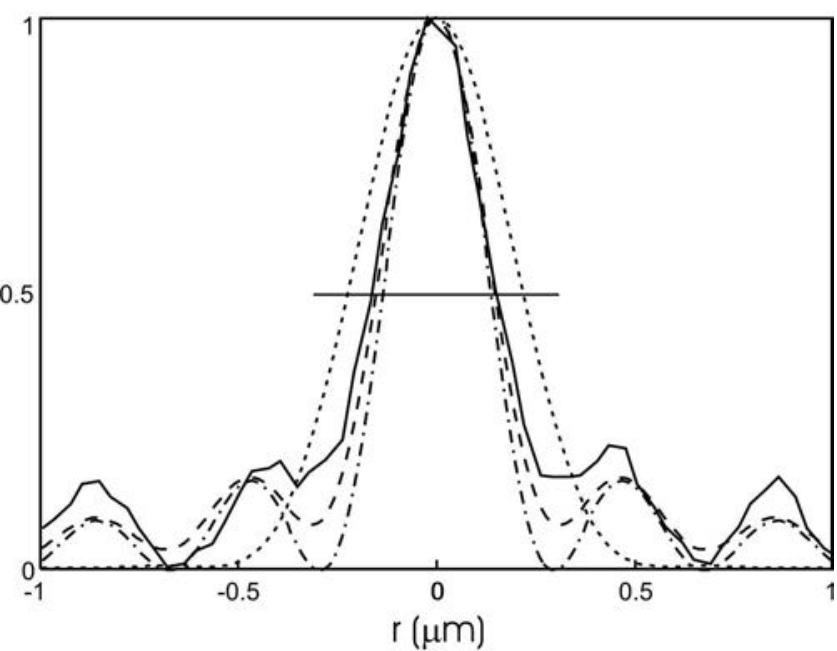

(d)

Fig. 3. (a) Intensity of the CPFB at focus (simulation); (b) transverse intensity of the CPBB (simulation); (c) map of the CPBB intensity (experiments). Image size, $2 \mu \mathrm{m}$ $\times 2 \mu \mathrm{m}$. (d) Profiles of (a) (dotted curve), (b) (dashed curve), (c) (solid curve), and of the smallest mark (dashed-dotted curve, simulation).

The spot size for the CPBB and the mark size exhibit almost the same value of $0.6 \lambda(300 \mathrm{~nm})$. It is 1.42 -fold smaller than the spot size of the CPFB. The difference between the spot sizes of the illuminating beams and the mark size are smaller than that for radial polarization. This is because circular polarization optimizes the focusing process for the lowest values of NA. ${ }^{3}$ According to Eq. (1), the mark size should be equal to $0.54 \lambda$, as for radial polarization. Because of convolution effects, the collecting local probe is assumed to broaden the central spot in the image.
Whatever the spot size of the incident light beam, we see that the mark size is minimized to a constant value that is slightly higher than the one predicted by Eq. (1). The small discrepancy between theory and experiments is due to the fact that the samples used in this study cannot linearly restore the square modulus of the active field component. Finally, we can assume that patterning with a near-field virtual tip $^{18-20}$ (evanescent Bessel beam) with NA=1.6 and $\lambda=488 \mathrm{~nm}$ should lead to a mark size smaller than $110 \mathrm{~nm}$. Smaller patterns can be achieved by reducing $\lambda$ and/or increasing NA. Moreover, let us recall that in Bessel beams exhibiting nondiffractive properties, the high transverse confinement is associated with very large focal depths, which is particular interesting in data storage.

In conclusion, we have experimentally shown that, by taking advantage of the $\mathrm{PF}$ properties of some photoresists, it is possible to achieve the smallest marks that can be lithographically generated with focusing devices. This offers the possibility of maximizing the optical data storage capacity in far-field regime.

T. Grosjean's e-mail address is thierry.grosjean@ univ-fcomte.fr.

\section{References}

1. T. D. Milster, Opt. Photonics News 16(3), 28 (2005).

2. T. Grosjean, F. I. Baida, and D. Courjon, "Conical optics: the solution of confine light," Appl. Opt. (to be published).

3. T. Grosjean and D. Courjon, "Smallest focal spots," Opt. Commun. (to be published).

4. T. Grosjean and D. Courjon, Opt. Express 14, 2203 (2006).

5. Y. Gilbert, R. Bachelot, P. Royer, A. Bouhelier, G. P. Wiederrecht, and L. Novotny, Opt. Lett. 31, 613 (2006).

6. L. E. Helseth, Opt. Commun. 191, 161 (2001).

7. R. M. Herman and T. A. Wiggins, J. Opt. Soc. Am. A 8, 932 (1991).

8. G. Scott and N. McArdle, Opt. Eng. 31, 2640 (1992).

9. R. Piron, E. Toussaere, D. Josse, and J. Zyss, Appl. Phys. Lett. 77, 2461 (2000).

10. P. Rochon, E. Batalla, and A. Natansohn, Appl. Phys. Lett. 66, 136 (1995).

11. D. Y. Kim, S. K. Triparthy, L. Li, and J. Kumar, Appl. Phys. Lett. 66, 1166 (1995).

12. T. Grosjean, D. Courjon, and M. Spajer, Opt. Commun. 203, 1 (2002).

13. K. Karraï and R. D. Grober, Ultramicroscopy 61, 197 (1995).

14. T. Grosjean and D. Courjon, Phys. Rev. E 67, 46611 (2003).

15. K. S. Youngworth and T. G. Brown, Opt. Express 7, 77 (2000).

16. Z. Bouchal and M. Olivik, J. Mod. Opt. 42, 1555 (1995).

17. Ph. Prêtre, L.-M. Wu, A. Knoesen, and J. D. Swalen, J. Opt. Soc. Am. A 15, 359 (1998).

18. T. Grosjean, D. Courjon, and D. Van Labeke J. Microsc. 210, 319 (2003).

19. E. Descrovi, V. Paeder, L. Vaccaro, and H.-P. Herzig, Opt. Express 13, 7017 (2005).

20. Q. Zhan, Opt. Lett. 31, 1726 (2006). 